| Revista: Ciencia, Tecnología y Política | Año 1 Número 1 | www.revistas.unlp.edu.ar/CTyP |

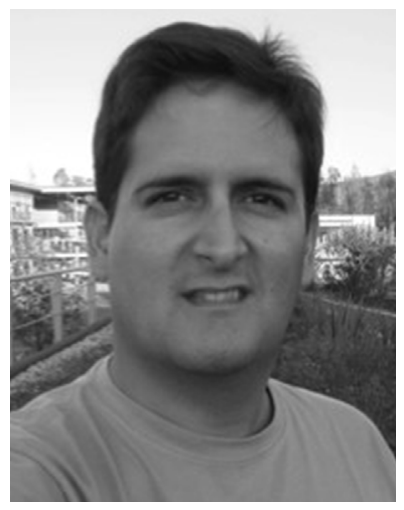

Juan Emilio Sala

Dr. en Ciencias Biológicas

Instituto de Biología de

Organismos Marinos

(IBIOMAR-CONICET),

Laboratorio de Problemáticas

Socio-Ambientales (FHCS)

Universidad Nacional de la

Patagonia San Juan Bosco,

Puerto Madryn, Chubut

juansala@cenpat-conicet.gob.ar

\title{
Pampa Azul: el mar como territorio
}

Resumen: Según un estudio prospectivo realizado entre 2013y 2015 porel Ministerio de Ciencia, Tecnología e Innovación Productiva, el aporte actual del sector marítimo al Producto Bruto Interno (PBI) del país es de sólo 1,5\% (concentrado en la actividad pesquera). Pero se estima que en 2035 podría alcanzar entre el 10 y el $15 \%$ del PBI si se realizara una mayor inversión en investigación, innovación tecnológica y desarrollo productivo. En este contexto nace la iniciativa Pampa Azul, que representa una política de Estado de gran envergadura para instalar al Mar Argentino, por primera vez, en la agenda de desarrollo de nuestro país. En este trabajo se analizan las principales características de esta iniciativa y particularmente la Ley № 27.167 (PROMAR), promulgada en 2015, que establece un presupuesto mínimo de 250 millones de pesos anuales para llevarla adelante. Asimismo se muestra la situación actual de Pampa Azul en el contexto de cambio de signo y de políticas de Gobierno Nacional.

Palabras claves: Pampa Azul; Mar Argentino; Soberanía; Recursos Naturales; Desarrollo Tecnológico; Investigación Oceánica y Marina.

\section{Pampa Azul: The sea like territory}

Abstract: According to a prospective study carried out between 2013 and 2015 by the Argentinean Ministry of Science, Technology and Productive Innovation, the current contribution of the maritime sector to the Gross Domestic Product (GDP) of our country is only $1.5 \%$ (concentrated in fishing activity). But it was estimated that in 2035 between 10 and $15 \%$ of GDP could be achieved if more investment is made in research, technological innovation and productive development. In this context, the Pampa Azul initiative represents a large-scale State policy to install the Argentine Sea, for the first time, in the development agenda of our country. This paper analyzes the main characteristics of this initiative and particularly the Law $\mathrm{N}^{\circ}$ 27.167 (PROMAR), promulgated in 2015, which establishes a minimum of 250 million pesos per year to carry it forward. It also shows the current situation of Pampa Azul initiative in the context of the change of signand politics of the National Government.

Keywords: Pampa Azul; Argentine Sea; Sovereignty; Natural Resources; Technological Development; Oceanic and Marine Research. 


\section{Introducción}

Los océanos cubren el $71 \%$ de la superficie de la Tierra y están vinculados a los medios de vida de nuestra especie de muchas maneras. Desde su papel en la modulación del clima hasta la forma en que proporcionan una variedad de beneficios socio-económicos, culturales y ambientales, los océanos -y sus marescontribuyen en gran medida al bienestar humano (WWF, 2015).

A partir de un relevamiento reciente se pone en evidencia el nivel de dependencia concreta de la humanidad en relación a los océanos y mares, y de su situación global actual, en términos de degradación, pudiéndose mencionar los siguientes ejemplos: 1) casi 3.000 millones de personas (es decir, casi la mitad de la población humana) dependen del pescado como fuente principal de proteínas; 2) la pesca y la acuicultura aseguran los medios de subsistencia del 10-12\% de la población mundial; 3) el 60\% de la población mundial vive a menos de 100 km de la costa; 4) las poblaciones de vertebrados marinos han disminuido aprox. 50\% entre 1970 y 2012; 5) las poblaciones de especies de peces comercialmente importantes se han reducido a la mitad, y algunas de las especies más importantes han experimentado disminuciones aún mayores; 6) casi el 30\% de las poblaciones de peces marinos están sobreexplotadas en todo el mundo; 7) más de 5 billones de piezas de plástico que pesan un total de más de 250.000 toneladas se encuentran en el mar. Sin embargo, los océanos globales generan beneficios económicos por el valor de al menos US\$2,5 billones por año (WWF, 2015). Paradójicamente, sólo el 3,4\% del océano está protegido o al menos bajo algún tipo de legislación ambiental, y aún menor es el porcentaje de territorio marino efectivamente manejado (WWF, 2015).

Para enfrentar estos desafíos y buscar comprender mejor el clima y los ecosistemas oceánico-marinos, así como los impactos y vulnerabilidades provocadas por las actividades humanas, se ha establecido un sistema para el monitoreo continuo y a largo plazo de observaciones oceánicas y/o marinas: el Sistema Global de Observaciones Oceánicas (GOOS por su sigla en inglés'). El GOOS es quien coordina, a escala planetaria, las observaciones en torno al océano mundial -y los mares que lo componen- enfocándose en tres ejes fundamentales: clima, salud del océano y servicios en tiempo real (e.g. pronósticos de las condiciones climáticas, entre muchos otros).

El GOOS es un programa ejecutado por la Comisión Oceanográfica Intergubernamental (IOC, por su sigla en inglés) de la Organización de las Naciones Unidas para la Educación, la Ciencia y la Cultura (UNESCO, por sus siglas en inglés), organismo especializado de las Naciones Unidas (ONU), pero su éxito radica en las contribuciones coordinadas de diversas personas y organizaciones de todo el mundo, contando con quince iniciativas de observación oceánico-marinas a lo largo y ancho del globo. Si bien esta red cubre buena parte de los mares de mayor importancia (en sentido amplio), gran parte del Mar Argentino permanece por fuera de su órbita ${ }^{2}$, resultando estratégico crear un programa similar para investigar y monitorear en tiempo real esta parte indisoluble e indispensable de nuestro territorio soberano.

\footnotetext{
'Más información en: http://www.goosocean.org/ (8 de Junio de 2018).

2 Ver: http://www.goosocean.org/index.php?option=com_content\&view=article\&id=83\&ltemid=121 (8 de Junio de 2018).
} 


\section{Pampa Azul: un paso hacia la incorporación (efectiva) del mar al territorio nacional}

Con la firme convicción enfocada en la búsqueda sistemática de ampliación y fortalecimiento de nuestra soberanía nacional, y a través del aprovechamiento y puesta en valor de los recursos científicos y tecnológicos propios, la ex-Presidenta Cristina Fernández de Kirchner lanza en abril de 2014 el programa Pampa $\mathrm{Azul}^{3}$, iniciativa de investigación científica estratégica enfocada en el Mar Argentino 4 . Esta decisión, constituye una verdadera política de Estado pensada a 10 años, cuyo espíritu se resume en su lema: "El conocimiento científico al servicio de la soberanía nacional". La iniciativa Pampa Azul (PA) es la primera acción nacional desarrollada a nivel interministerial con foco en nuestro mar territorial: participan los Ministerios de Ciencia, Tecnología e Innovación Productiva (MinCyT), Relaciones Exteriores y Culto, Agricultura, Ganadería y Pesca (hoy Agroindustria), Turismo, Defensa, Seguridad, Medio Ambiente y Desarrollo Sustentable ${ }^{5}$. Sus principales objetivos buscan profundizar el conocimiento científico como fundamento de las políticas de conservación y manejo de los recursos naturales; promover innovaciones tecnológicas aplicables a la explotación sustentable de los recursos naturales y al desarrollo de las industrias vinculadas al mar; fortalecer la conciencia marítima de la sociedad argentina y respaldar con información y presencia científica la soberanía de nuestro país en el área del Atlántico Sur.

La iniciativa hace foco en cinco áreas geográficas prioritarias, sobre la base de sus características oceanográficas, la relevancia de sus ecosistemas y el impacto (potencial o consumado) de las actividades antrópicas. Estas son: (1) Banco Namuncurá/Burdwood (reciente Área Marina Protegida cercana a Tierra del Fuego); (2) Agujero Azul (en el talud continental); (3) golfo San Jorge (Chubut y Santa Cruz); (4) áreas marinas sub-antárticas (incluyen Islas Georgias y Sandwich del Sur); y (5) los estuarios bonaerenses. Bajo este marco, ya se han realizado varias campañas de investigación, aunque principalmente concentradas sobre las áreas del Banco Namuncurá-Burdwood y del golfo San Jorge, no obstante sin fondos propios de la iniciativa (i.e. dichas campañas se han financiado con partidas originadas de otra fuente de financiamiento distinta a la establecida por la Ley PROMAR, debilitando la institucionalización y el sostenimiento definitivo de la iniciativa Pampa Azul).

\footnotetext{
${ }^{3}$ El nombre que lleva la iniciativa intenta, metafóricamente, equiparar la importancia que tiene para la Argentina la "Pampa Verde" y húmeda, apostando a la consumación de una "Pampa Azul" productiva y sustentable, donde el país y su sociedad dejen de vivir de espaldas al mar, parte indisoluble de su territorio. Como nota de color se puede contar que el ex-Gobernador de la Provincia de Chubut, Martín Buzzi, se autoproclama como el creador tanto del nombre como de la idea original que motivó la iniciativa Pampa Azul.

${ }^{4}$ Más información en: http://www.pampazul.gob.ar/ (8 de Junio de 2018).

${ }^{5}$ Se encuentran involucradas en dicha iniciativa las siguientes instituciones: el Consejo Nacional de Investigaciones Científicas y Técnicas (CONICET); la Dirección Nacional del Antártico (DNA); la Comisión Nacional de Actividades Espaciales (CONAE); el Instituto Nacional de Investigación y Desarrollo Pesquero (INIDEP); el Servicio de Hidrografía Naval (SHN); la Prefectura Naval Argentina; el Centro Austral de Investigaciones Científicas (CADIC-CONICET); el Centro Nacional Patagónico (CENPAT-CONICET); el Instituto Argentino de Oceanografía (IADO-CONICET/UNS); el Instituto Investigaciones Marinas y Costeras (IIMyC-CONICET); el Centro de Investigaciones del Mar y la Atmósfera (CIMA-CONICET/UBA); el Instituto de Biología Marina y Pesquera Almirante Storni (UNCO); la Universidad Nacional del Comahue; la Universidad Nacional de la Patagonia San Juan Bosco; la Universidad Nacional de Tierra del Fuego, Antártida e Islas del Atlántico Sur; la Universidad Nacional de la Patagonia Austral; la Universidad Nacional de Mar del Plata; la Universidad Nacional del Sur; la Universidad Nacional de La Plata; y la Universidad de Buenos Aires.
} 
Dado que el PA es un programa muy costoso (e.g. se estima que un día de campaña oceanográfica requiere de aprox. 80.000 dólares únicamente para hacer frente a la cuestión operativa [Sala et al., 2017]), en 2015 el Gobierno Nacional preparó y envió al Congreso de la Nación, para su posterior aprobación (29 de julio de 2015), el Proyecto de Ley № 27.167 (PROMAR), mediante la cual se crea el Programa Nacional de Investigación e Innovación Productiva en Espacios Marítimos Argentinos. Posteriormente, se designa al MinCyT como la Autoridad de Aplicación (vía Decreto Nacional Nº604/2016). La norma, promulgada en septiembre del 2015, consta de ocho artículos dentro de los cuales se desarrollan los lineamientos generales y específicos para el fortalecimiento de la presencia soberana del país en el Mar Argentino, pero además tiene como unos de sus principales objetivos atender a la iniciativa PA. Un aspecto central es que a través de esta ley se crea, también, el Fondo Nacional para la Investigación e Innovación Productiva de los Espacios Marítimos Argentinos (FONIPROMA); integrado por las partidas que destine el Presupuesto General de la Administración Pública Nacional y/o con la afectación del crédito presupuestario de las partidas que reasigne el Poder Ejecutivo; y cuyo monto inicial "no podrá ser inferior a los 250 millones de pesos", que provendrán de subsidios, préstamos u otros aportes (donaciones, etc.).

Entre los aportes científicos que derivan del programa Pampa Azul en pos del apuntalamiento y ampliación de la soberanía nacional se pueden destacar: el incremento en el conocimiento de base de nuestro mar y sus ecosistemas (considerados como altamente productivos a nivel global); la generación y el manejo de nuevas Áreas Marinas Protegidas; el desarrollo de capacidades tecnológicas vinculadas al aprovechamiento sustentable de los recursos (biológicos o energéticos) de nuestra plataforma continental; la sustitución de importaciones; la generación de puestos de trabajo genuino y calificado, y la ocupación activa y productiva (en el más amplio de los sentidos) de nuestro territorio marino. En este sentido, y según un estudio prospectivo realizado entre 2013 y 2015 por el MinCyT, y publicado en 2017, titulado "Horizontes estratégicos para el Mar Argentino", nuestro territorio marino posee un potencial de ingresos para el período 2016-2035 que se calcula en alrededor de 220.000 millones de dólares. El aporte actual del sector marítimo al PBI representa sólo el 1,5\% debido, principalmente, a la actividad pesquera; pero las estimaciones del estudio elevan esa cifra hasta llegar a un aporte potencial de entre el 10 y el 15\% del PBI, si se incrementara adecuadamente la inversión en investigación, innovación tecnológica y desarrollo productivo.

Más allá de este invalorable potencial, existe una consecuencia aún más directa -y profunda- de los resultados de una iniciativa como ésta sobre la soberanía nacional. El ejemplo paradigmático lo constituye el estratégico pedido de ampliación de nuestra Zona Económica Exclusiva (ZEE), de 200 a 350 millas náuticas, que realizó en 2009 la Comisión Nacional de Límite de la Plataforma Continental Argentina (COPLA), a través de la Cancillería, ante la Comisión del Límite Exterior de la Plataforma Continental (CLPC), un órgano científico integrado por 21 expertos internacionales de reconocido prestigio y creado por la Convención sobre Derecho del Mar (CONVEMAR), organismo de la ONU encargado de resolver estas cuestiones ${ }^{6}$.

\footnotetext{
${ }^{6}$ Vale destacar que el llamado de la CLPC de 2009, para que los diversos estados nación que quisieran reclamar una ampliación en el límite exterior de sus plataformas continentales, el último, y la ONU denegaría reclamos posteriores al mismo.
} 


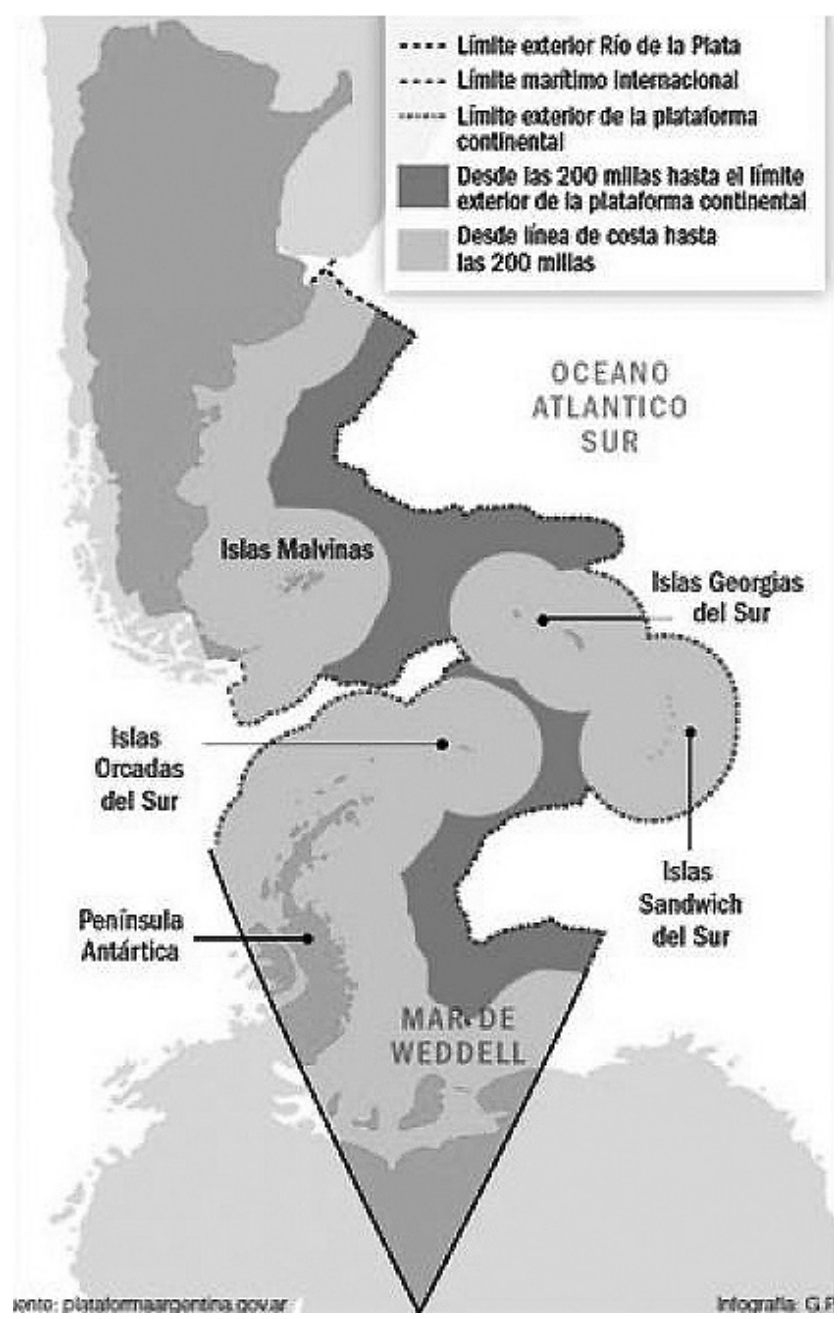

Figura 1. Mapa bicontinental de la República Argentina donde se indican los límites exteriores de la Plataforma Continental Argentina. El área en gris claro se corresponde con el límite exterior de las 200 millas náuticas, el cual fue recientemente extendido hasta la milla 350 (área en gris oscuro), gracias a la presentación realizada por la Cancillería Nacional en 2009 y aprobada con la CONVEMAR (ONU) en 2016. Fuente: http://plataformaargentina.gov.ar.
El 11 marzo de 2016 el pedido de ampliación fue aceptado por unanimidad por la CLPC y a través de ello la Argentina pasó a contar con 1.700 .000 $\mathrm{km}^{2}$ de territorio marino nuevo para administrar? sumándose así a los $4.800 .000 \mathrm{~km}^{2}$ que ya conformaban su ZEE (ver Figura 1). Sobran las palabras respecto al significado concreto -y simbólico- que esta decisión de la ONU tiene ante nuestro reclamo histórico de soberanía sobre las Islas Malvinas e Islas del Atlántico Sur. Lo que interesa destacar acá es que el pedido de la COPLA (que en honor a la verdad se venía trabajando desde 1996), fue acompañado, como justificación, por trece tomos de cuantiosa y sólida información científica, de todo tipo, colectada y sintetizada a través de expertos geodestas, hidrógrafos, geólogos, geofísicos, cartógrafos, oceanógrafos, expertos en sistemas de información geográfica, abogados y expertos en derecho internacional. Sin esta valiosísima información el pedido nunca hubiera sido aceptado. Se trata de una muestra contundente de poner a trabajar los recursos propios de nuestro sistema científico tecnológico de cara a la ampliación y consolidación de la soberanía nacional, en el más amplio y profundo de los sentidos.

La combinación de medidas que contribuyen a la ampliación y consolidación del Estado y sus capacidades, a la ampliación de derechos sociales e individuales y al incremento de la soberanía, es quizás el legado más importante dejado por la experiencia de los últimos tres gobiernos nacionales (2003-2015). En este marco el PA y el trabajo desarrollado por nuestra comunidad científica para la COVEMAR son un ejemplo de lo que podemos Ilamar Ciencia Nacional: poner el conocimiento, los recursos y la capacidad científico tecnológica del país en proyectos liderados por el Estado destinados a resolver necesidades nacionales y regionales de carácter estratégico, social o económico, en un marco de desarrollo e inclusión social.

\footnotetext{
${ }^{7}$ Sin embargo, la resolución de la CLPC le otorga únicamente derechos soberanos a nuestro país sobre los recursos existentes sobre y debajo el lecho marino del nuevo territorio (i.e. minerales, hidrocarburos y especies sedentarias, como langostas y mejillones, entre otros recursos por explotar), excluyendo así los recursos contenidos en la columna de agua.
} 


\section{Presupuesto nacional devengado para Ciencia y Técnica}

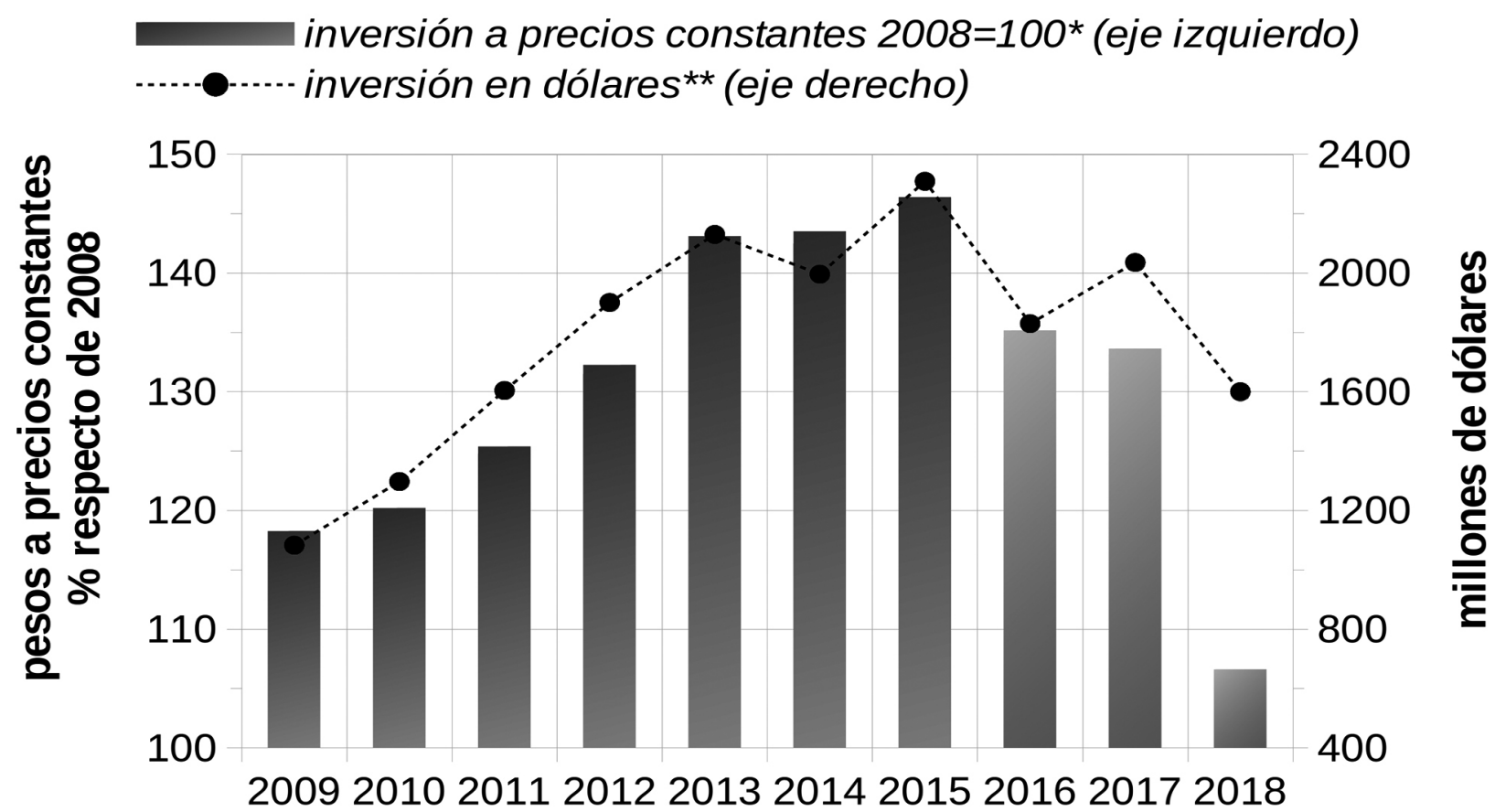

Figura 2. Evolución del presupuesto nacional devengado para el sector de ciencia y tecnología entre 2009 y 2018. Gentileza de "Defendamos la Ciencia Argentina” (@DefLaCienArg).

*Fuentes de IPC empleado: 2008-2016, a partir de IPC elaborado por "Cosas que Pasan" http://ow.ly/95u530fitiF:INDEC (26.1\%); 2018 estimaciones a partir de Relevamiento de Expectativas de Mercado de BCRA publicado en marzo de 2018 (23.0\%)

${ }^{* *}$ calculado utilizando la cotización media anual obtenida a partir del Tipo de Cambio Nominal Promedio Mensual informado por BCRA; para el año 2015 no se incluyó en el promedio la cotización alcanzada luego de la devaluación de diciembre. Para el 2018 el promedio sólo cuenta con datos hasta marzo.

Nota: valores para 2017 actualizados al 18/03/2018; para 2018 estimados suponiendo igual \% de ejecución que el \% promedio entre 2016 y 2017, sobre presupuesto actualizado al 20/03/2018.

\section{La agenda del ajuste, la readecuación del Estado y el endeudamiento externo}

Dicen los que saben que no hay mejor forma de leer, aunque sea entre líneas, cuál es la lista de prioridades, el modelo de desarrollo y el rumbo de país elegido por una gestión política presidencial que la confección de la Ley de Presupuesto General de la Administración Pública Nacional (conocida como "ley de leyes", por obvias razones). Así, desde octubre de 2016, momento en que fuera presentado en el Congreso de la Nación el presupuesto para 2017 (primer presupuesto presentado por el actual gobierno), el área de Ciencia y Técnica (CyT) vio reducido muy fuertemente su participación en el mismo, lo que motivó una andanada de medidas de fuerza a nivel nacional que culminaron con la toma del MinCyT, allá por diciembre de 2016, entre el brindis y el pan dulce (Rodríguez Mega, 2016; Román, 2016, 2017). Sin buscar profundizar mucho en la serie de conflictos desatados y la fuerte reacción organizada y colectiva de resistencia que despertó en la comunidad académica (e.g. la creación de un Frente Federal de Ciencia y Universidad), vale detenerse brevemente en la pérdida irrefrenable de soberanía científico-tecnológica que se viene dando -e intensificando- desde el 10 de diciembre de 2015. Bajo la agenda del ajuste, la reestructuración del Estado y el endeudamiento externo, se inició un camino de desmembramiento sistemático y políticamente planificado de las instituciones de ciencia y técnica. 
Un análisis todavía más interesante -y fiable- para conocer los intereses y prioridades respecto de las políticas públicas de una gestión es evaluar los niveles de ejecución de dichas partidas presupuestarias. El Gobierno Nacional ha publicado los datos actualizados sobre la ejecución presupuestaria del 2016 y $2017^{8}$ los que denotan una considerable jerarquización de los montos destinados al pago de la deuda pública (se incrementó casi 250\% en el acumulado bianual, respecto de 2015) en perjuicio de sectores como salud, educación, cultura y ciencia y tecnología. Más específicamente, el presupuesto devengado (ejecutado) para CyT experimentó una caída (real) del 20\% bianual (respecto a 2015, incluyendo las recomposiciones que se dan durante cada ejercicio). Incluso, las partidas previstas para CyT en 2018 experimentan un fuerte retroceso (en relación porcentual sobre el presupuesto nacional total) respecto de último trienio (ver Figura 2). La retracción presupuestaria es tan solo una parte de un plan sistemático de paralización y vaciamiento de todos los proyectos y programas de desarrollos tecnológicos estratégicos nacionales, en donde el sector CyT tenía un rol preponderante (e.g. ARSAT 3 y 4, Tronador II, Atucha III, Fabricaciones Militares, FADeA, Programa de Producción Pública de Medicamentos, entre otros). Esto es parte de una política mucho más amplia de desindustrialización con la concomitante pérdida de soberanía -de todo tipo- que implica. Cómo ya se dijo más arriba, la iniciativa PA, por sus características, es muy costosa en términos económicos y por ello se pensó en la Ley PROMAR, la cual crea el FONIPROMA. A través de esta ley se le garantizaban fondos propios al Programa Pampa Azul, y demás actividades enmarcadas en la ley, con un piso de $\$ 250.000 .000$. Si bien este monto fijo en pesos tiene poco sentido práctico, ya que si se actualizan por inflación los 250 millones de pesos asignados en 2015 arrojaría un número cada vez más abultado, lo cierto es que esa suma inicial de base hubiera permitido dar inicio real a la mayor parte de las líneas de investigación y desarrollo que la iniciativa PA preveía. Sin embargo, la gestión de la Alianza Cambiemos, destinó en el Presupuesto General de la Administración Pública Nacional de 2017 tan solo 4,7 millones de pesos a la Ley PROMAR (Stefani, 2016), incumpliéndola de facto. Ahora, lo más penoso es que además de incumplir la ley, sólo se le asigne a la iniciativa el $4 \%$ del presupuesto basal estipulado en la misma, siendo que con ello no se puede costear ni siquiera el combustible de los buques oceanográficos para realizar las campañas.

Otro grave problema es que no se conoce el presupuesto real devengado para el año 2016 y 2017, y mucho menos se conoce cuál es el monto real con el que cuenta la Ley № 27.167 (PROMAR) para el ejercicio en curso. Estas últimas cuestiones motivaron que el Diputado Nacional por la Provincia de Buenos Aires (Bloque Unidad Ciudadana-FpV), Dr. Roberto Salvarezza (ex-Presidente del CONICET y uno de los principales impulsores de la iniciativa PA), eleve un pedido de informe al Poder Ejecutivo Nacional, a través del MinCyT, para lograr conocer, fehacientemente, los montos asignados y ejecutados durante 2016 y 2017; cuál es el nivel de ejecución y actividades desarrolladas por la Ley PROMAR durante el 2018; y cuáles son los planes de acción estratégica que diseñó el Consejo Administrativo del PROMAR para los años 2016, 2017 y 2018.

\footnotetext{
${ }^{8}$ Ver: www.datos.gob.ar/dataset/ejecucion-presupuestaria-de-la-administracion-publica-nacional (8 /06/18)
} 


\section{Conclusiones}

La iniciativa Pampa Azul fue concebida como una política nacional a diez años, transversal a una gran cantidad de ministerios, con una ley marco que la acompañaba, y con una cantidad de objetivos multipropósito de carácter netamente estratégico.

Por primera vez en décadas, la Argentina definió una política de Estado destinada a integrar el espacio marítimo al desarrollo nacional. Para lo cual, mediante una normativa ad hoc estableció objetivos y un plan de acción con recursos económicos, técnicos y humanos.

Por lo tanto, el potencial de un programa como Pampa Azul, tanto en el plano económico como en el geopolítico es, sin dudas, de una gran envergadura. Lamentablemente, sólo algunas de las acciones previstas comenzaron a ejecutarse en las áreas marítimas identificadas como estratégicas.

En el marco de la reestructuración macro-sistémica que está llevando adelante la Alianza Cambiemos con el Estado, su rol y funciones, Pampa Azul está siendo víctima de un duro ataque, tanto en términos económicos como de gestión. La asignación presupuestaria 2017 alcanza tan solo un 4\% del monto nominal previsto en 2015, lo cual es una muestra evidente de la intención de paralizar o desactivar el programa. Estas políticas de "ajuste" neoliberal dañan particularmente a los emprendimientos científico-tecnológicos que apuntan al desarrollo nacional autónomo y soberano. En el caso de Pampa Azul amenazan, incluso, la propia subsistencia del programa, en la medida que se trata de una iniciativa muy joven y, por tanto, poco consolidada.

La breve pero significativa historia de Pampa Azul es una nueva evidencia de que el proyecto neoliberal, actualmente en ejecución en Argentina, tiene entre sus más importantes consecuencias -y objetivos velados-, la disminución -y en algunos casos, la destrucción- de toda iniciativa que contribuya a la soberanía nacional en los planos político, económico y tecno-científico.

\section{Referencias}

Rodríguez Mega, E. (2016) Argentina's scientists engulfed in budget crisis. Science DOI: 10.1126/science.aal0388

Román, V. (2016) Argentina president's first budget angers scientists. Nature DOI:10.1038/nature.2016.21013

Román, V. (2017) Argentina's researchers occupy science ministry. Nature DOl:10.1038/nature.2017.21242

Sala, J.E., Pisoni, J.P. y Quintana, F. (2017) Three-dimensional temperature fields of the North Patagonian Sea recorded by Magellanic penguins as biological sampling platforms. Estuarine, Coastal and Shelf Science, DOI: 10.1016/j.ecss.2017.03.021.

Stefani, F.D. (2016) Magnitud y consecuencias del recorte presupuestario del MINCYT en comparación con 2016. Disponible en: http://www.nano.df.uba.ar/wordpress/wp-content/uploads/Magnitud-y-Consecuencias-del-recorte-al-MINCYT-con-respecto-a-2016.pdf. (18/6/18)

WWF (2015) Living Blue Planet Report. Species, habitats and human well-being. Tanzer, J., Phua, C., Lawrence, A., Gonzales, A., Roxburgh, T. and P. Gamblin (Eds). WWF, Gland, Suiza. 